\title{
THE USE OF SPATIAL TERMS “NEAR”, "VERY NEAR, “NEXT TO”, "SIDE BY SIDE AND "NEARBY" IN THE DESCRIPTIONS OF SPATIAL CONFIGURATIONS
}

\author{
Maria Engracinda dos Santos Ferreira ${ }^{1}$ - ORCID: 0000-0002-7746-4626 \\ Luciene Stamato Delazari ${ }^{1}$ - ORCID: 0000-0003-0018-085X \\ 1 Universidade Federal do Paraná, Programa de Pós-Graduação em Ciências Geodésicas, \\ Curitiba, Paraná, Brasil \\ E-mail: maria_ufrrj@yahoo.com.br \\ E-mail: luciene@ufpr.br
}

Received in $28^{\text {th }}$ May 2018

Accepted in $27^{\text {th }}$ February 2019

\section{Abstract:}

Spatial relations are words used to describe the existing relationships between elements present in environments. In the Natural Language, that is, that of the representations present geographical spatial organizations often seen in the daily lives of individuals, the user when reporting their location, uses a significant amount of spatial relations. Consequently, one has the difficulty of defining which of these words are suited to be used in spatial descriptions, in the sense of transmitting spatial information in a clear and precise way. Aiming to foster research in this field, an experiment was carried out with volunteers natives Brazilian Portuguese language in which the use of five spatial relations denoting distances in qualitative terms was sought. The results obtained showed that, although they present similar understandings, the incorrect use of one of these spatial relations can transmit to the receiver of the message an erroneous spatial information. In addition, it was observed a tendency in choosing a specific spatial relation as the distance between the reference elements increases with each other.

Keywords: Spatial Relations; Spatial Descriptions; Spatial terms; Natural Language. 


\section{Introduction}

When describing the strategies needed to reach a specific location or report the spatial configuration of an environment, the individual performs the spatial description task. The purpose of these descriptions is to identify objects or events in a specific environment or to provide information from a specific, unknown region to the user receiving the information (Hall et al. 2015; Stock 2010; Herskovits 1985). Such descriptions are commonplace in people's daily life and occur naturally, since in dealing with abstract notions human beings choose to reflect using spatial concepts - as a consequence of their ability to reason spatially. This explains why the Natural Language (NL) - a system of signals that allows interaction and communication among human beings - is so linked to spatial concepts and, therefore, to make such concepts so present in the day by day (Kracht 2002).

The spatial descriptions use spatial terms to describe the connection existent among elements presents inthe world and their arrangement in the environment. The selected elements, called landmarks (Li et al. 2011; Herskovits 1985), are features chosen based on their characteristics that make them more salient in relation to others (Li et al. 2011; Winter et al. 2005; Raubal and Winter 2002; Lynch 1960). In addition, landmarks should be recognized and recalled by users in an easy and unambiguous way (Sorrows and Hirtle 1999; Presson and Montello 1988; Lynch 1960). The elements indicated as landmarks provide crucial information about the environment that enable the observer to perform both navigation and self-locating tasks (Raubal and Winter 2002; Golledge et al. 2000; Sorrows and Hirtle 1999; Presson and Montello 1988; Lynch 1960). The spatial terms, called as spatial relation, have the characteristic of relating the elements present in the visual scenes described (Li et al. 2011; Herskovits 1985).

The branches of science and engineering that work with spatial information search the formal understanding of spatial relations (Egenhofer and Franzosa 1991), since these are the basis for many selections made by users when querying geographic information systems (GIS). Despite the use of spatial relations in spatial descriptions, systems that seek to process and represent such spatial descriptions, often not understood effectively their meaning. This is because spatial relations in NL are rich, imprecise, unstructured, but with qualitative quality whereas in GIS, they are quantitative, structured and precise. Therefore, studies of spatial relations aim at understanding how users use these words in their daily lives. In other words, such studies search for understanding how to use spatial relations for describing spatial configurations, to then, implement them in the GIS systems (Kracht 2002; Mark, 2000; Egenhofer and Mark 1995).

Shariff et al. (1998) showed the influence caused by the meaning of the terms used as spatial relations. The authors believe that the nature of the elements presented in the environment influences how users describe and understand spatial relations. When dealing with the relations of proximity, Schockaert et al. (2005) showed in his work that cognitive distortions can occur due to the scale of the elements used as references in spatial descriptions. Fisher and Orf (1991) sought to define individual characteristics that justify the choice of users by the use of one or another spatial relation in the evaluation of distances between elements. Their results did not allow categorizing the individuals according to their choices between the spatial relations "near" and "close". Moreover, the study revealed the loss of the spatial meaning of these relations due to the function and name of the reference elements. Foley and Cohen (1984) pointed to errors in estimating distances between objects and such errors increased as people became more familiar with the environment. The results of Gahegan (1995) indicated that changes in the 
perception of distances between elements occurs according to the reference element. All these results are in accordance with the studies of Golledge et al. (1975, apud Fisher and Orf 1991), in which the authors prove the inconsistency of the estimates of distances between objects because the perception is exclusive to each individual.

Despite the use of spatial relations in descriptions of visual scenes, the systems which seek to process and represent such descriptions, often do not understand their meaning effectively. This is because spatial relations in NL are rich, imprecise, unstructured, and of qualitative quality, whereas in GIS they are quantitative, structured, and accurate. Thus, the spatial relations in the GIS do not portray the same meaning when compared to those used by the users in a spatial description through the NL (Zhang et al. 2013; Stock 2010; Mark and Egenhofer 1995; Egenhofer and Franzosa 1991). In addition, the fact that NL is dynamic, complex and spatial relations are vague (Stock 2010) makes it difficult to implement systems capable of understanding it. Another issue is the versatility of the spatial terms used as spatial relations. That is, only one spatial relation can describe different spatial configurations or still, several spatial relations describe the same environmental configuration (Hall and Jones 2008).

Therefore, this work aims to understand the use of spatial relations "near", "very near", "next to", side by side" and "nearby" as well as to indicate the spatial relations appropriate to the spatial configurations presented, since the understanding of such relations is important because reflect the way in which the observed environment is understood, related and described by the users through NL. Therefore, it is intended to help the implementation of these spatial relations in NL processing and representation systems, such as the application, object of study of the project "Where I am?", in which this work is inserted. In the mentioned project, it is sought to convert a spatial description of a place into a geographical location. Thus, it is of utmost importance to understand the use of spatial relations in scenarios experienced by users daily, and if possible, to define the word that describes how features presented in the environment are connected to each other.

\section{Methodology}

Spatial relations are spatial terms used for describing the relationship among features existed at environmental. Therefore, it is a fundamental question in obtaining geographic information. For this reason, this work studied such terms used by users when performing the description of a location. The experiment consisted of drawings representing possible geographical spatial organizations, in which the user chose a spatial description that he/she considered appropriate for the presented spatial configuration. For this, a questionnaire was elaborated, in which schematic drawings representative of possible geographical configurations were described through five locative expressions. The methodology adopted for this research is presented below.

\subsection{Participants}

The participants were stratified according to 'his/her age and it was not required an identification, so it was an anonymous experiment. This methodology was adopted since 
anonymity allows greater freedom in the responses and also provides more security because the answers are not identified (Marconi and Lakatos 2003). All data were provided by the participants after they signed a consent form, agreeing to the use of the information to conduct the research. Thus, a sample of 73 volunteers answered the requested questionnaire.

\subsection{Description of the Method}

In the methodological proposal of this research, the users' test was established using a qualitative approach. It was decided to use this method, since it aims to generate and modify initial conceptions about a specific topic of study (Suchan and Brewer 2000). For this purpose, a structured questionnaire was elaborated, in which the participants had to selected one of the alternatives provided. The questionnaire was produced on a digital platform (Google Forms) and distributed through social networks, aiming to reach more people. The experiment was available to receive responses from September to October 2017.

In the experiment, it was used schematic drawings for representing spatial configurations present in real environments. Shariff et al. (1998) argue that in spatial relations or spatial knowledge acquisition or navigation experiments, normally the schematic drawings used do not present similarities with maps. According to the authors, users do not interpret schematic drawings as real elements present in physical space. Instead, users understood the images only as dots, lines, and polygons. For this reason, it was sought in this experiment a spatial arrangement of elements in such a way as to represent scenarios experienced by users in everyday life.

The experiment was carried out in the Brazilian Portuguese language using a form available online, in which, at the first moment, the users declared to accept or refuse their participation, through the consent term. Later, explanations were provided about the experiment. At this time, it was assured the users understanding in relation to four issues:

1. The drawn figures, called features A and B, represent landmarks.

2. The representations present geographical spatial configurations often seen in the daily lives of individuals.

3. To each scenario will be shown five possible spatial descriptions.

4. The user should read and opt for the description that he/she judges representative for the relationship between landmarks for each schematic figure.

The fourth and last step consisted in the presentation of the schematic figures, which denote the spatial configurations, with five descriptions. As the experiment was carried out in the Brazilian Portuguese language, for this article the spatial relations used in the descriptions of the schematic drawings, "perto", "muito perto, "próximo", "ao lado" and "junto", were translated into "near", "very near", "next to", "side by side" and "nearby". It was asked to the user to choose only one sentence that represents the spatial configuration of the elements for each scenario. Figure 1 is a scenario created with four possible spatial descriptions. 


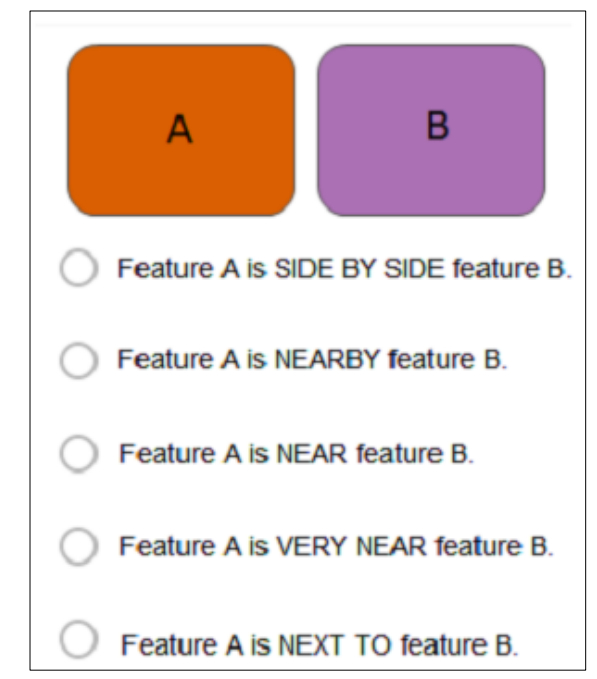

Figure 1: Proposed schematic drawing with the presence of two structural elements called of "A" and "B feelings and the local phrases developed for the scenario.

It can be seen in Figure 1 that the only distinction between features, called $A$ and $B$, is its color. Different colors do not imply that one feature is more salient than the other, but rather the user's intention to interpret as two different elements. It is necessary to clarify that, the characteristics that make the elements in potential landmarks were not considered in the choice of the scenarios for the accomplishment of this test. That is means, we were only worried about the more common scenes in the daily life of the individuals, since the experiment aims to understand the choice of the relation to the spatial configurations commonly found.

The spatial arrangement of the elements in the environments daily experienced by individuals motivated the creation of the scenarios. Figure 2 shows an example, in which the elements chosen as landmarks were school and hotel. These elements were represented in the schematic drawing in the Figure 1.

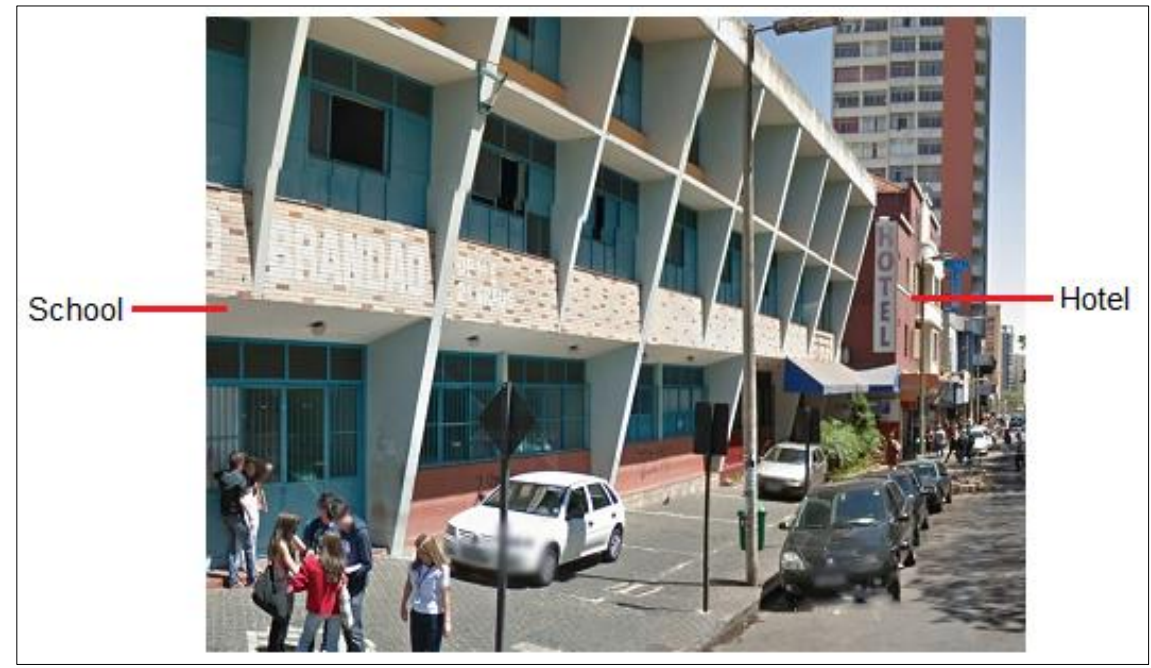

Figure 2: Space configuration found in real environments used as a model for the schematic design. 
In order to carry out the experiment of this article, the users had to visualize four representative scenarios of spatial configurations experienced by them in their daily life, as shown in Figure 3. As previously shown, the features $A$ and $B$ are elements present in the environment, such as schools, buildings, houses, squares, described by users as landmarks. The spatial configurations can be seen in Figure 3.

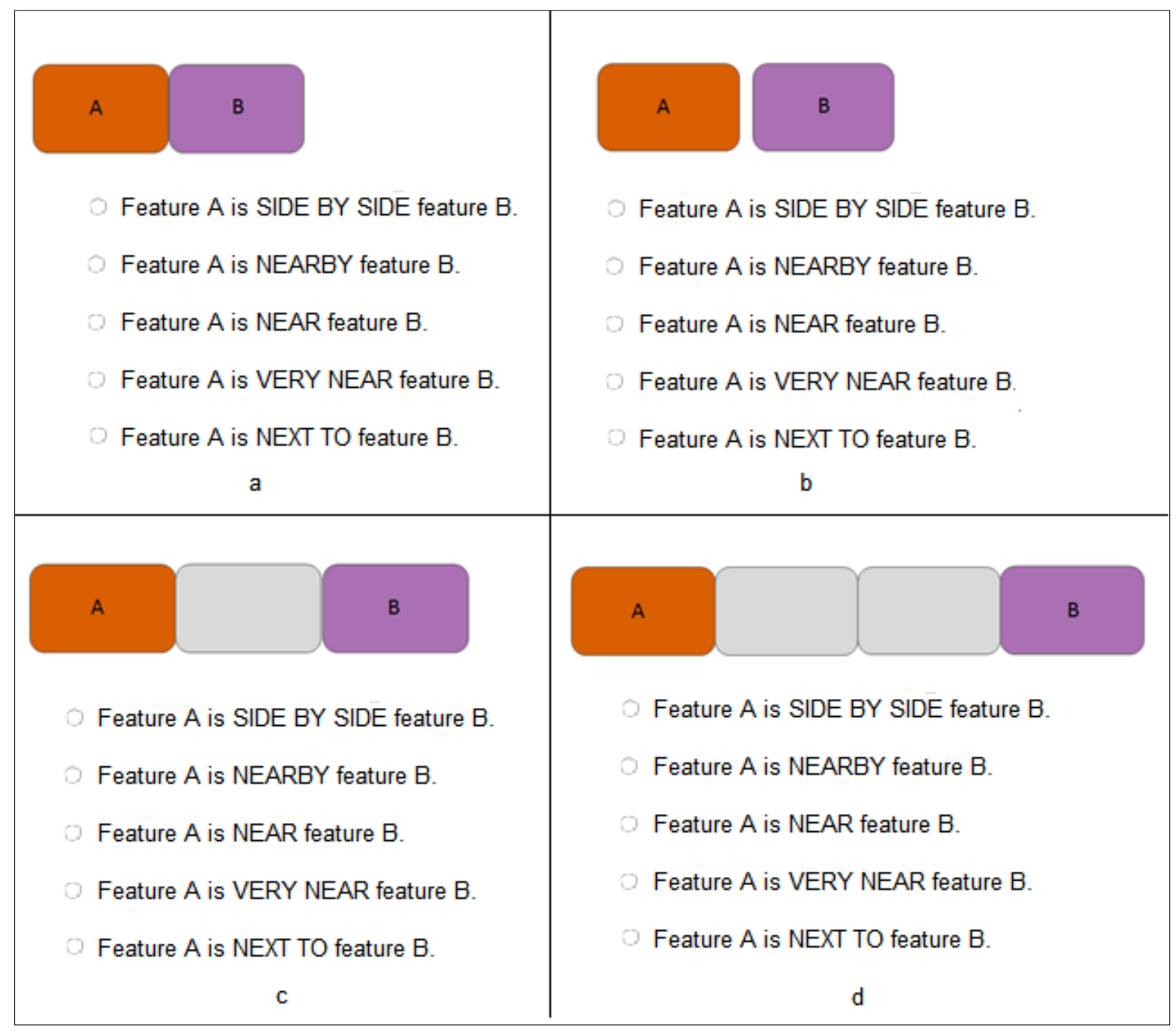

Figure 3: Representative schematic drawings of four possible space configurations.

Figure 3 presents the scenarios presented for the users. The four spatial configurations, presented in Figure 3, were spatially characterized using the same spatial descriptions: "Feature $A$ is side by side feature B", "Feature $A$ is near feature B", "Feature A is very near feature B", "Feature $A$ is next to feature $B$, "Feature $A$ is nearby feature $B$ ". The locative expressions were the same for the four scenarios presented in Figure 3, and the experiment was designed in a way that allowed users to choose only one locative expression per scenario. In addition, users had the possibility to return to the scenario already answered, review their answers and modify them, if they thought necessary. It is important to point out that the users visualized only one scene at a time and that the elaborated spatial configurations intended to convey to the user an increase in distance between features $A$ and $B$ insofar as new elements in the composition of the scenarios are introduced. 


\section{Results and Discussions}

The experiment reported in this article was proposed with the aim of understanding the use of spatial relations "near", "very near", "next to", "side by side" and "nearby", and consequently, to optimize the use of such words in the task of spatial description. The spatial relations addressed are associated with distances in a qualitative aspect rather than metric terms and such concepts are still poorly defined (Mark 2000). Fisher and Orf (1991) reinforce this weighting. These authors performed experiments with spatial relations that denote distance in qualitative terms, and such experiments did not bring conclusions that can relate the concepts of these spatial relations used in NL to geographic queries. With the objective of collaborating with this issue, this article sought to specify the adequate spatial relation, according to the preference of the users who answered the questionnaire, for the four spatial configurations.

\subsection{Participants}

The experiment obtained a sample of 73 responses during the period that was available to users. Regarding the age group, it was not possible to make observations confronting the spatial relations chosen with the age of the users. This was due to uneven sampling, since the experiment obtained 26 and 27 users in the age groups of 20 to 29 years and 30 to 39 years, respectively. Whereas only 4, 10, and 6 users were stratified at age 19 or younger, ages 40-49 and older than 50 , in that order. Figure 4 shows the percentages obtained from participants for each age group.

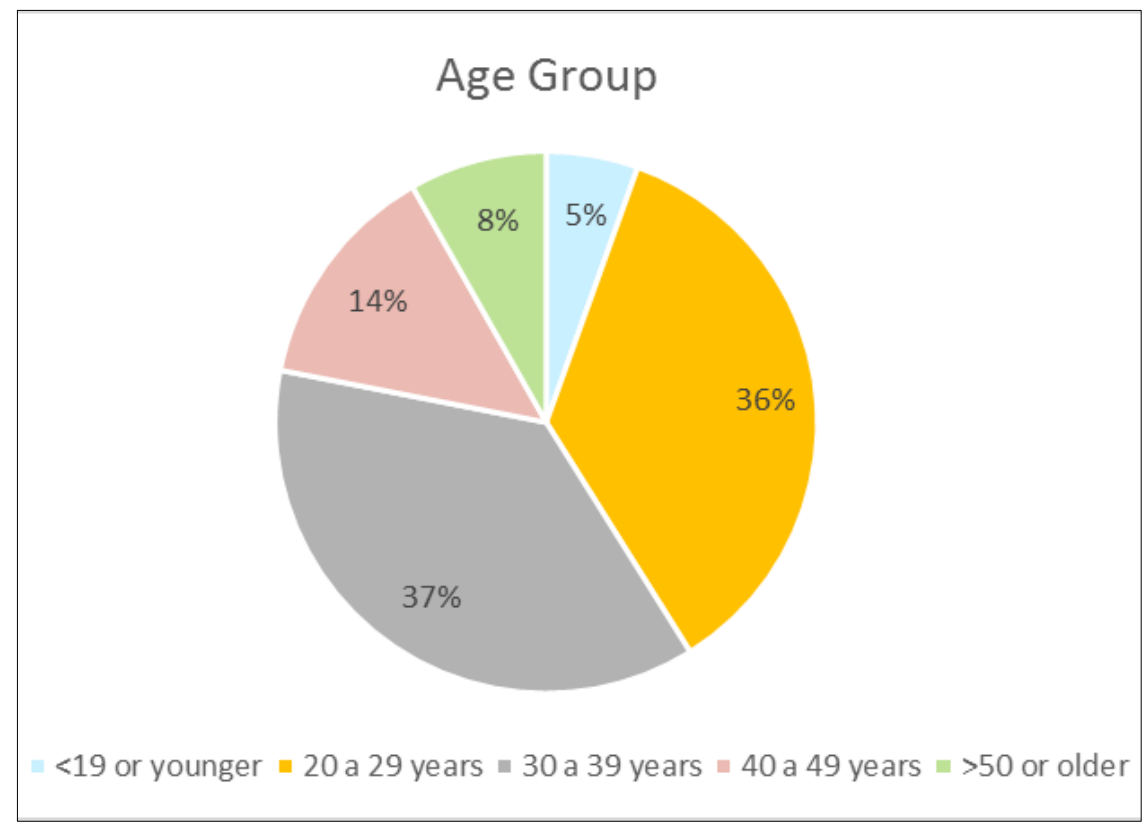

Figure 4: Age group of the experiment participants. 


\subsection{Obtained data}

This work is an attempt to understand the use of a set of spatial relations ("near", "very near", "next to", "side by side" and "nearby") in describing spatial configurations experienced in individuals. Consequently, according to the choices made by the participants of the experiment, it was sought to indicate the appropriate spatial relation for each scenario. Figure 5 shows the values obtained for the spatial relationships studied in the four scenarios presented in Figure 3 . With the obtained data it was possible to make considerations about the most representative spatial relations for the spatial configurations approached, according to the preference of the participants who carried out this experiment.

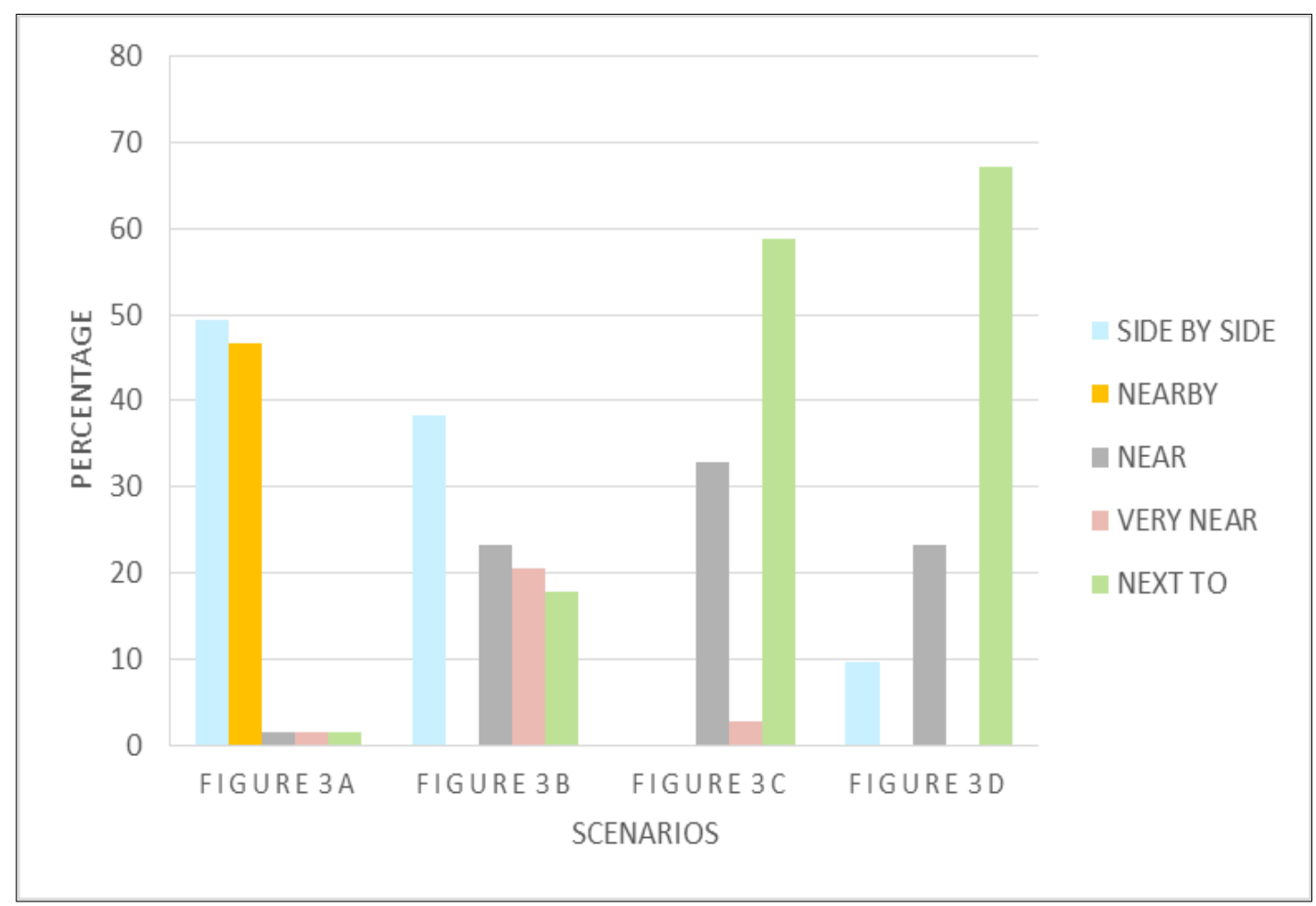

Figure 5: Use of spatial relations by scenario (as shown in Figure 3).

For the spatial configuration presented by the schematic drawing of Figure 3a, all the spatial relations of this experiment, "side by side", "near", "very near", "next to", "nearby". Despite this, attention should be paid to the fact that the spatial relations "side by side" and "nearby" covered $95.6 \%$ of the users" choices, being $46.6 \%$ for "nearby" and $49.3 \%$ for "side by side".

From the four proposed conjunctures, the one illustrated by Figure $3 b$ presented the greater balance in relation to the identification or choice of an adequate spatial relation to describe the represented spatial configuration. However, the spatial relation "nearby" was not selected in describing this scenario. The spatial relation "side by side" was chosen by $38.4 \%$ of the volunteers, followed by "near" spatial relation with 23.3\%, "very near" with $20.5 \%$ and "next to" with $17.8 \%$. The percentages shows that, unlike the previous situation, this configuration presented similar proportions, having a greater prominence for the spatial relation "side by side". 
In relation to Figure 3c, the "next to" spatial relation was chosen by $58.9 \%$ of the users, followed by "near" with $32.9 \%$, "side by side" with $5.5 \%$ and "very near" with $2.7 \%$. As in the previous scenario, users did not use the spatial relation "nearby". In the last scenario presented in Figure $3 d$, the "next to" spatial relation had $67.1 \%$ of users' preference, followed by the "near" with $23.3 \%$ and "side by side" with $9.6 \%$. For this scenario, users did not choose spatial relations "very near" and "nearby".

As can be seen in the Figure 5, there is a tendency to choose the "near" and "next to" spatial relations as features A and B distance themselves. In other words, the number of users opting for such relationships increases considerably as the scenarios advance. Figure 6 shows the propensity to use such spatial relationships separately.

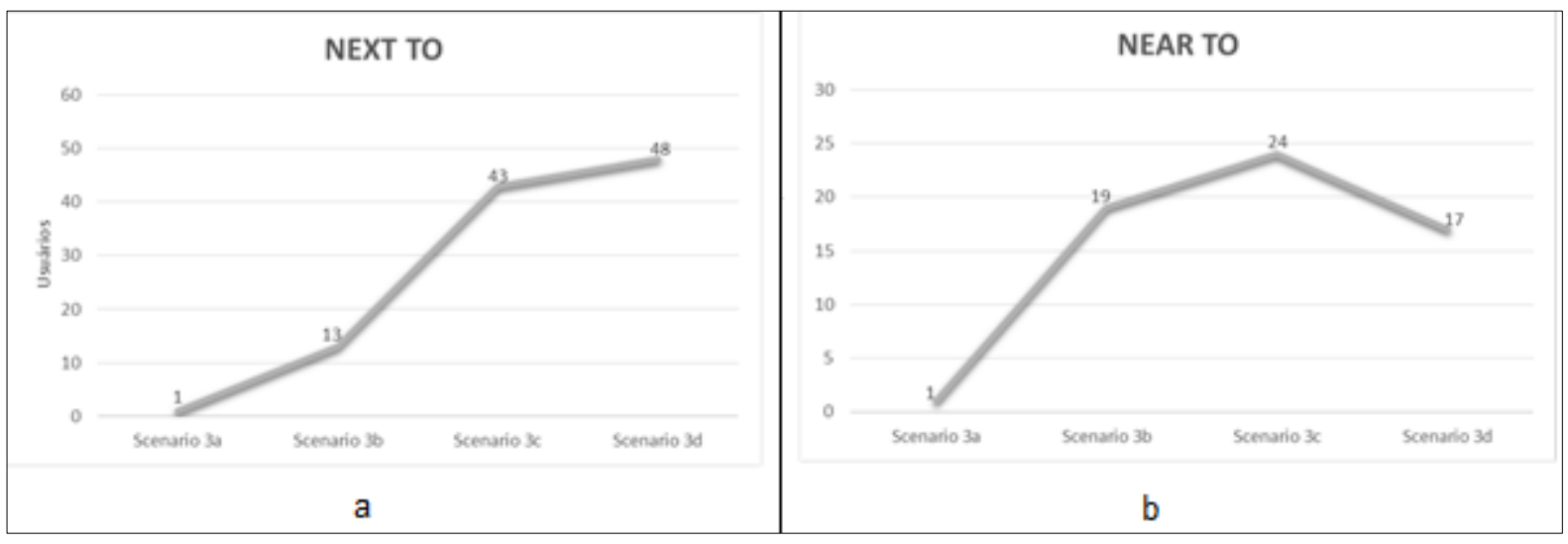

Figure 6: Use of spatial relations "near" and "next to" by scenario.

From the figure above, it was noticed that the use of the spatial relation "next to" increases as the distance between elements $A$ and B increases. The same happens with the spatial relationship "near to", although there was a significant drop in its use when scenario changes from $3 c$ to $3 d$. Confronting this result with the work of Freundschuh and Blades (2012), in which the authors have conducted experiments to evaluate the development of the spatial concept of spatial relations "near" and "next to" (equivalent in Portuguese of "perto" and "próximo") from childhood to adulthood, it was noticed the incompatibility of the conclusions in the relations mentioned, although in different language. These authors concluded that with increasing age, to represent smaller distances between objects, the users used the spatial relation "next to". In other words, when users had to locate certain objects using the "next to" spatial relation, the distance between these objects was smaller than when using the "near" spatial relation.

The research presented in this paper only worked with adult users and in it, it was verified a tendency of the majority of the users for the use of the "next to" relation as the distance between elements used as reference increases with each other (Figure 6), different from the results obtained by Freundschuh and Blades (2012). Figure 6b shows a decrease in the use of the "near to" as the distance between the elements $A$ and $B$ increases. This result differs from those obtained by Freundschuh and Blades (2012) since in the study the spatial relationship "near to" was chosen by adults to represent distances greater than the distances represented by the spatial relationship "next to". Probably, this divergence can be explained by the cultural, social and linguistic differences existing between the individuals who carried out the experiment of Freundschuh and Blades (2012) and those who contributed to the research presented in this 
article. These factors contribute to the choice of spatial relations used in the descriptions of space. This is because they influence the way in which the environment is perceived by the individual and, consequently, in the acquisition of the knowledge (Stock 2010; Montello 2002; Levinson 1996).

Another point observed was that, although the five spatial relations addressed in this research have similar linguistic meanings, the meaning of the spatial description might change if the spatial relation is misused (Mark et al. 1989). This is the case of the spatial relation "nearby". Although it is present in all spatial configurations the users only choose it in the spatial configuration of scenario 1, as shown in Figure 7.

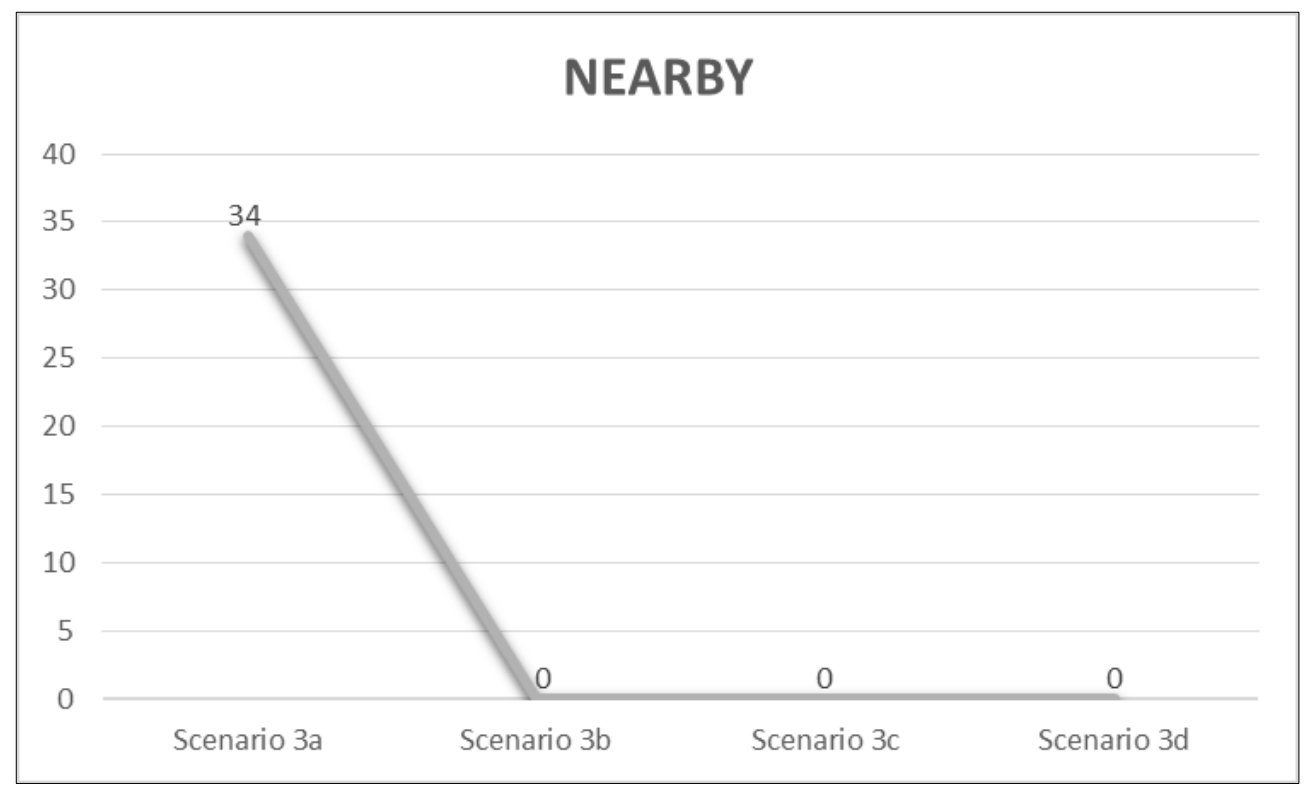

Figure 7: Use of spatial relation "nearby" by scenario.

Therefore, it can be concluded that when using spatial relation "nearby" for represent any of the other scenarios, the understanding of the information received by the user will be done in the wrong way, since the above-mentioned spatial relationship was chosen only to describe scenario 3a. To ensure the use of the correct spatial relation to describe the spatial position of objects, and that such information is transmitted and assimilated in a clear and precise manner, are issues that contribute to the difficulty of modeling NL processing and representation programs (Herskovits 1985).

The results obtained also show the versatility of spatial terms used as spatial relations (Hall and Jones 2008). In other words, the same spatial relation is used to describe different configurations between elements or the opposite, different spatial relation to reporting the positioning of the elements present in the same scenario. Examples of the first situation are the "near" and "next to" spatial relations, present in the users' choices in all scenarios. Given that, in scenarios 1 and 2, the choice was small when compared to the other spatial relations, but the users used them. The results obtained for scenario 2 corroborate with the affirmation of the possibility of different spatial relations reporting the same environmental configuration. This because it was the scenario that presented a balance in relation to the choice of spatial relations. 
That is, there is no preference for a specific spatial relation. This may mean that any of the four spatial relations chosen in this scenario can be used to convey the spatial information.

\section{Conclusions}

The objective of this research was to understand the use of spatial relations that denote distances in qualitative terms in spatial descriptions, in an attempt to show which of them is suitable for each of the four spatial configurations proposed, according to the choice of participants. The results indicated that there is a user preference for the "next to" and "near" spatial relations as the landmarks distance between them. The spatial relation "next to" was chosen by the majority of users when there are one or more elements (scenarios presented in Figure $3 \mathrm{c}$ and $3 \mathrm{~d}$ ) between landmarks, that is, when there is a greater distance between them.

The experiment also showed that although the spatial relationships used erroneously cause similar understandings for users, it does not imply that the choice of any of them denotes the same spatial information. In opposite, the misuse of a word to describe the relationship between elements can convey misleading information. Still, the versatility of spatial relationships was evidenced, that is, a spatial relation can describe different spatial configurations, as well as the opposite, a single configuration can be reported using different spatial relations. This characteristic hampered to this experiment to indicate the adequate spatial relation to describe each scenario. This objective was not reached since only the spatial relationship "nearby" had its exclusive use for scenario presented in Figure 3a. The results obtained contribute to the search of the spatial terms of distances aiming the implementation of such words in a processing and representation system of $\mathrm{NL}$, that will be implemented by the project "Where Am I?". In order to contribute with future work should be done to solidify the results obtained, so it is suggested to work only with the "next to" and "near" spatial relations.

\section{Acknowledgement}

The authors would like to thank the CNPq for the financial support (grant 310312/2017-5 and $459300 / 2014-8)$.

\section{Author's contribution}

Both authors contribute equally. 


\section{References}

Egenhofer, M.J. and Franzosa, R.D., 1991. Point-set topological spatial relations. International Journal of Geographical Information Systems, 5(2), pp.161-174.

Egenhofer, M.J. and Mark, D.M., 1995. Modelling conceptual neighbourhoods of topological lineregion relations. International Journal of Geographical Information Science, 9(5), pp.555-565.

Fisher, P.F. and Orf, T.M., 1991. An investigation of the meaning of near and close on a university campus. Computers, Environment and Urban Systems, 15(1-2), pp.23-35.

Foley, J.E. and Cohen, A.J., 1984. Mental mapping of a megastructure. Canadian Journal of Psychology/Revue canadienne de psychologie, 38(3), pp.440-453.

Freundschuh, S. and Blades, M., 2012. The Cognitive Development of the Spatial Concepts NEXT, NEAR, AWAY and FAR. Cognitive and Linguistic Aspects of Geographic Space, pp.43-62.

Gahegan, M., 1995. Proximity operators for qualitative spatial reasoning. Lecture Notes in Computer Science (including subseries Lecture Notes in Artificial Intelligence and Lecture Notes in Bioinformatics), 988, pp.31-44.

Golledge, R.G. et al., 2000. Cognitive maps, spatial abilities and human wayfinding. Geographical Review of Japan, Series B, 73(2), pp.93-104.

Hall, M.M. and Jones, C.B., 2008. Quantifying spatial prepositions. Proceedings of the 16th ACM SIGSPATIAL international conference on Advances in geographic information systems GIS 08, (October 2016), p.1.

Hall, M.M., Jones, C.B. and Smart, P., 2015. Spatial Natural Language Generation for Location Description in Photo Captions. In Proceedings of the 12th International Conference on Spatial Information Theory - COSIT. New York, pp. 196-223 SRC-GoogleScholar FG-0.

Herskovits, A., 1985. Semantics and Pragmtics of Locative Expressions. Cognitive Science, 9(3), pp.341-378.

Kracht, M., 2002. On the Semantics of Locatives. Linguistics and Philosophy, 25(1995), pp.157232.

Levinson, S.C., 1996. Frames of reference and Molyneux" s question : Crosslinguistic evidence. Language and Space, pp.109-169.

$\mathrm{Li}, \mathrm{X}$. et al., 2011. Describing spatial locations from perception and memory: The influence of intrinsic axes on reference object selection. Journal of Memory and Language, 65(2), pp.222236.

Lynch, K., 1960. The imagem of the city. Cambridge: The MIT press.

Marconi, M. and Lakatos, E., 2003. Fundamentos de metodologia científica. Editora Atlas. Sao Paulo. pp. 195-199.

Mark, D., 2000. Ontological Foundations for Geographic Information Science. Engineering, pp.18.

Mark, D.M. and Egenhofer, M.J., 1995. Topology of Prototypical Spatial Relations Between Lines and Regions in English and Spanish 1, (March), pp.245-254. 
Mark, D.M. and Frank, A.U., 1989. Concepts of space and spatial language. In Proceedings, Auto Carta 9. pp. 538-556.

Montello, D.R., 2002. Cognitive Map-Design Research in the Twentieth Century: Theoretical and Empirical Approaches. Cartography and Geographic Information Science, 29(3), pp.283-304.

Presson, C.C. and Montello, D.R., 1988. Points of reference in spatial cognition: Stalking the elusive landmark. British Journal of Developmental Psychology, 6, pp.378-381.

Raubal, M. and Winter, S., 2002. Enriching Wayfinding Instructions with Local Landmarks. Proceedings of the Second International Conference on Geographic Information Science, 2478(1), pp.243-259.

Schockaert, S., De Cock, M. and Kerre, E.E., 2005. Automatic acquisition of fuzzy footprints. Lecture Notes in Computer Science (including subseries Lecture Notes in Artificial Intelligence and Lecture Notes in Bioinformatics), 3762 LNCS, pp.1077-1086.

Shariff, A.R.B.M., Egenhofer, M.J. and Mark, D.M., 1998. Natural-Language Spatial Relations Between Linear and Areal Objects: The Topology and Metric of English- Language Terms *. Mark International Journal of Geographical Information Science, 12(3), pp.215-246.

Sorrows, M.E. and Hirtle, S.C., 1999. The Nature of Landmarks for Real and Electronic Spaces. In COSIT - Spatial Information Theory. Cognitive and Computational Foundations of Geographic Information Science. Stade, pp. 37-50.

Stock, K., 2010. Describing Spatial Relations using Informal Semantics. In Proceedings of GIS Research UK. London, pp. 14-16.

Suchan, T.A. \& Brewer, C.A., 2000. Qualitative Methods for Research on Mapmaking and Map Use. Professional Geographer, 52(1), pp.145-154.

Winter, S., Raubal, M. and Nothegger, C., 2005. Focalizing measures of salience for wayfinding. Map-based Mobile Services: Theories, Methods and Implementations, pp.125-139.

Zhang, C., Zhang, X. and Du, C., 2013. The relevance of spatial relation terms and geographical feature types. Lecture Notes in Computer Science (including subseries Lecture Notes in Artificial Intelligence and Lecture Notes in Bioinformatics), 7769 LNAI(40971231), pp.47-56. 